\title{
Measurement of $K^{*}(892)^{0}$ and $K^{0}$ mesons in $\mathrm{Al}+\mathrm{Al}$ collisions at $1.9 A \mathrm{GeV}$
}

\author{
X. Lopez,, , N. Herrmann,${ }^{2}$ K. Piasecki, ${ }^{2,3}$ A. Andronic,${ }^{4}$ V. Barret,${ }^{1}$ Z. Basrak,${ }^{5}$ N. Bastid,${ }^{1}$ \\ M.L. Benabderrahmane,${ }^{2}$ P. Buehler, ${ }^{6}$ M. Cargnelli, ${ }^{6}$ R. Čaplar, ${ }^{5}$ P. Crochet,${ }^{1}$ P. Dupieux,${ }^{1}$ \\ M. Dželalija,${ }^{7}$ L. Fabbietti, ${ }^{8}$ I. Fijał-Kirejczyk,${ }^{3}$ Z. Fodor, ${ }^{9}$ P. Gasik, ${ }^{3}$ I. Gašparić,${ }^{5}$ Y. Grishkin, ${ }^{10}$ \\ O.N. Hartmann, ${ }^{6}$ K.D. Hildenbrand, ${ }^{4}$ B. Hong, ${ }^{11}$ T.I. Kang, ${ }^{11}$ J. Kecskemeti, ${ }^{9}$ M. Kirejczyk, ${ }^{3}$ \\ Y.J. Kim, ${ }^{4}$ M. Kiš,${ }^{4,5}$ P. Koczon,${ }^{4}$ M. Korolija,${ }^{5}$ R. Kotte, ${ }^{12}$ A. Lebedev,${ }^{10}$ Y. Leifels,${ }^{4}$ V. Manko,${ }^{13}$ \\ J. Marton, ${ }^{6}$ T. Matulewicz,${ }^{3}$ M. Merschmeyer,${ }^{2}$ W. Neubert,${ }^{12}$ D. Pelte,${ }^{2}$ M. Petrovici, ${ }^{14}$ F. Rami, ${ }^{15}$ \\ W. Reisdorf, ${ }^{4}$ M.S. Ryu,${ }^{11}$ P. Schmidt,${ }^{6}$ A. Schüttauf, ${ }^{4}$ Z. Seres,${ }^{9}$ B. Sikora,${ }^{3}$ K.S. Sim,${ }^{11}$ V. Simion,${ }^{14}$ \\ K. Siwek-Wilczyńska, ${ }^{3}$ V. Smolyankin, ${ }^{10}$ K. Suzuki,${ }^{6}$ Z. Tyminski,${ }^{3}$ P. Wagner,${ }^{15}$ E. Widmann, ${ }^{6}$ \\ K. Wiśniewski, ${ }^{3}$ Z.G. Xiao, ${ }^{16}$ I. Yushmanov, ${ }^{13}$ X.Y. Zhang, ${ }^{16}$ A. Zhilin, ${ }^{10}$ and J. Zmeskal ${ }^{6}$ \\ (FOPI Collaboration) \\ P. Kienle ${ }^{6,8}$ and T. Yamazaki ${ }^{17}$ \\ ${ }^{1}$ Clermont Université, Université Blaise Pascal, CNRS/IN2P3, Laboratoire \\ de Physique Corpusculaire, BP 10448, F-63000 Clermont-Ferrand, France \\ ${ }^{2}$ Physikalisches Institut der Universität Heidelberg, Heidelberg, Germany \\ ${ }^{3}$ Institute of Experimental Physics, University of Warsaw, Warsaw, Poland \\ ${ }^{4}$ Gesellschaft für Schwerionenforschung, Darmstadt, Germany \\ ${ }^{5}$ Ruđer Bošković Institute, Zagreb, Croatia \\ ${ }^{6}$ Stefan-Meyer-Institut für Subatomare Physik, Österreichische Akademie \\ der Wissenschaften, Boltzmanngasse 3, A-1090, Wien, Austria \\ ${ }^{7}$ University of Split, Split, Croatia \\ ${ }^{8}$ Physik Department, Technische Universität München, D-85748 Garching, Germany \\ ${ }^{9}$ KFKI Research Institute for Particle and Nuclear Physics, Budapest, Hungary \\ ${ }^{10}$ Institute for Theoretical and Experimental Physics, Moscow, Russia \\ ${ }^{11}$ Korea University, Seoul, Korea \\ ${ }^{12}$ Institut für Strahlenphysik, Forschungszentrum Dresden-Rossendorf, Dresden, Germany \\ ${ }^{13}$ Kurchatov Institute, Moscow, Russia \\ ${ }^{14}$ Institute for Nuclear Physics and Engineering, Bucharest, Romania \\ ${ }^{15}$ Institut Pluridisciplinaire Hubert Curien, In2P3/CNRS, and Université de Strasbourg, Strasbourg, France \\ ${ }^{16}$ Institute of Modern Physics, Chinese Academy of Sciences, Lanzhou, China \\ ${ }^{17}$ Heavy-Ion Nuclear Physics Laboratory, RIKEN, Wako, Saitama 351-0198, Japan
}

(Dated: October 30, 2018)

\begin{abstract}
New measurement of sub-threshold $K^{*}(892)^{0}$ and $K^{0}$ production is presented. The experimental data complete the measurement of strange particles produced in $\mathrm{Al}+\mathrm{Al}$ collisions at $1.9 \mathrm{~A}$ $\mathrm{GeV}$ measured with the FOPI detector at SIS/GSI. The $K^{*}(892)^{0} / K^{0}$ yield ratio is found to be $0.0315 \pm 0.006$ (stat.) \pm 0.012 (syst.) and is in good agreement with the UrQMD model prediction. These measurements provide information on in-medium cross section of $K^{+}-\pi^{-}$fusion which is the dominant process on sub-threshold $K^{*}(892)^{0}$ production.
\end{abstract}

PACS numbers: 25.75.-q, 25.75.Dw

The FOPI Collaboration has performed a high statistics experiment to study strangeness production in $\mathrm{Al}+\mathrm{Al}$ collisions at a beam kinetic energy of $1.9 \mathrm{~A} \mathrm{GeV}$. The collected data sample is large enough to enable for the first time the reconstruction of deep sub-threshold resonance production of $K^{*}(892)^{0}$. This measurement extends the data to four strange particle species reported in [1] with two strange resonances the $\Sigma(1385)$ and the $K^{*}(892)^{0}$, and two neutral strange particles, the $\Lambda$ and the $K^{0}$. Strange resonances such as $K^{*}(892)^{0}$ and $\Sigma(1385)$ are particularly interesting to probe the earlier stage of the collision and the subsequent evolution of the medium. Indeed, these resonances are produced at subthreshold energy and their productions stemmed mainly from the fusion of pions and $K^{+}(\Lambda)$ 2], the latest being produced at the beginning of the collision [3, 4]. In addition, $K^{*}(892)^{0}$ and $\Sigma(1385)$ have short life time (4 and $5 \mathrm{fm} / c$, respectively) [5]. The measurement of $K^{*}(892)^{0}$ and $K^{0}$ is detailed in the present letter and their yield ratio is compared to the predictions of the UrQMD transport model.

The experiment was performed at the Heavy-Ion Synchrotron SIS of the GSI in Darmstadt by using an $\mathrm{Al}$ beam of kinetic energy of $1.9 \mathrm{~A} \mathrm{GeV}$ on an $\mathrm{Al}$ target. The target thickness was $567 \mathrm{mg} / \mathrm{cm}^{2}$ and the average beam intensity was $8 \cdot 10^{5}$ ions $/ \mathrm{s}$. The FOPI detector is an azimuthally symmetric apparatus made of several sub-detectors which provide charge and/or mass determi- 
nation over nearly the full solid angle. The central part of the detector is placed in a super-conducting solenoid and consists of a Central Drift Chamber (CDC) surrounded by a plastic scintillators (Barrel) for time of flight measurements. The forward part is composed of two walls of plastic scintillators (PLAWA and ZDD) and another drift chamber (Helitron) placed inside the super-conducting solenoid. More details on the FOPI apparatus and its different sub-detectors can be found in $[6-8]$. The target was placed at $40 \mathrm{~cm}$ upstream of its nominal position in order to cover the whole backward hemisphere [9]. Events are selected according to their centrality which is determined with the charged particle multiplicity measured in the CDC and in the PLAWA. The results presented here correspond to central collisions $\left(\sigma_{g e o}=315 \mathrm{mb}\right)$ representing about $20 \%$ of the total geometrical cross section.

For the present analysis, the $K^{*}(892)^{0}$ resonance (called $K^{* 0}$ in the following) was reconstructed from its decay products in the channel $K^{+} \pi^{-}$(branching ratio $66 \%[5])$. Pions are measured in the $\operatorname{CDC}\left(23^{\circ}<\theta_{l a b}<\right.$ $114^{\circ}$ ) and are identified by their mass determined by the correlation between magnetic rigidity and specific energy loss. $K^{+}$are identified by matching the CDC and the surrounding ToF-barrel. This restricts the measured phase space of $K^{+}$to $32^{\circ}<\theta_{l a b}<57^{\circ}$. More details on $K^{+}$identification with the FOPI detector are provided in [10, 11]. Long-lived neutral strange particle like the $K_{S}^{0}$ are identified via their charged decay particles in the CDC. A complete description of the $K_{S}^{0}$ reconstruction method can be found in [9].

The measurement of $K^{* 0}$ corresponds to deep subthreshold production $(800 \mathrm{MeV})$ since the threshold energy to create $K^{* 0}$ in elementary reaction is $2.75 \mathrm{GeV}$. Because of its strong decay into $K^{+}$and $\pi^{-}$, the short life time of this resonance does not allow us to disentangle its decay vertex from the collision vertex. Therefore, the invariant mass analysis consists of correlating primary kaons and pions. The invariant mass plot of reconstructed $K^{* 0}$ is shown in Fig. 1. In order to enhance the signal-to-background ratio, a set of conditions was imposed:

(i) a cut on $K^{+}$momentum $\left(p^{l a b} \leq 0.68 \mathrm{GeV} /\right.$ c) is applied to reduce the contamination from fast pions and protons;

(ii) a cut on the $\pi^{-}$transverse momentum ( $p_{t}^{l a b}>$ $0.185 \mathrm{GeV} / \mathrm{c}$ ) is used to reject pions which are spiraling in the CDC;

(iii) a cut on the distance of closest approach (DCA) between a track and the collision vertex in the transverse plane $\left(\mathrm{DCA}_{K^{+} / \pi^{-}}<1 \mathrm{~cm}\right)$ was applied to suppress decay products outside the target;

(iv) a condition was imposed on the minimum number of activated wires forming a track (hits) in the CDC
(30 for $K^{+}$and 40 for $\pi^{-}$) for a higher quality of track reconstructions;

(v) the following cut was applied for the systematic study of the signal (see text for more details): $160^{\circ}<\theta_{K^{+}}^{*}+\theta_{\pi^{-}}^{*}<200^{\circ}, \theta^{*}$ being the angle between the momentum vector of a decay product in the reference frame of $K^{* 0}$ and the momentum vector of $K^{* 0}$ in the reference frame of the collision. Its relevance is described in [12.

The combinatorial background is obtained with the event-mixing method. The two decay particles $\left(K^{+}\right.$and $\pi^{-}$) are taken from two different events characterized by the same particle multiplicity in the CDC. In addition, the two events are aligned to the reaction plane in order to have the same reference system for both particles. The reaction plane is estimated event by event utilizing the standard transverse momentum procedure proposed in [13]. The combinatorial background was normalized on the left and right side of the nominal mass of the $K^{* 0}$. For either sides no difference was found on the signal characteristics (mean, width and counts). Figure 1 shows the normalization on the left side (hatched region in upper panel of Fig. 11). The shape of the resulting mixed-event background describes the combinatorial background and is indicated by dashed lines in Fig. 1 (upper panel) where the statistical error bars are smaller than the line thicknesses. After background subtraction

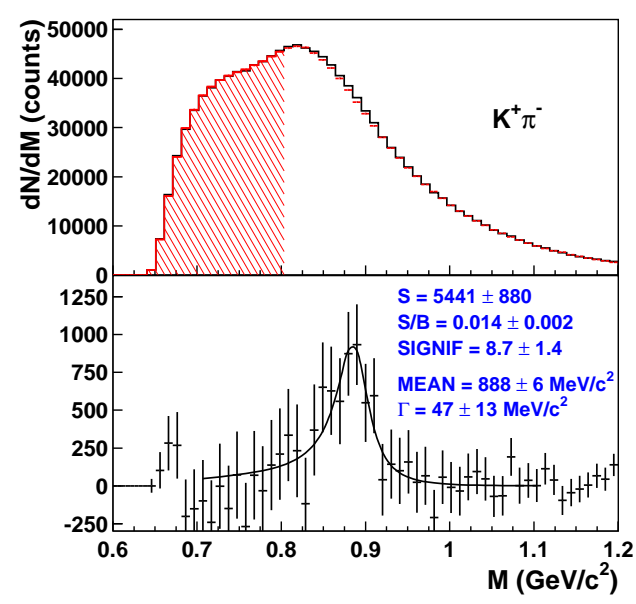

FIG. 1: (Color online) Invariant mass spectra of $K^{+} \pi^{-}$ pairs. The solid histogram and the dashed lines denote the data and the scaled mixed-event background, respectively (upper panel). The lower panel shows the signal after background subtraction. The following characteristics of the signal are shown: number of counts in the signal (S), signal-to-background ratio $(\mathrm{S} / \mathrm{B})$ and significance $(\mathrm{SIGNIF}=$ $S / \sqrt{S+B})$. The parameters extracted from the fit to the data (mean mass value $(\mathrm{MEAN})$ and the width $(\Gamma))$ are also reported.

(lower panel of Fig. 1), within the extracted width (47 
$\mathrm{MeV}$ ), about $5400 \mathrm{~K}^{* 0}$ are reconstructed from the analysis of 290 million events. The fitted function used to extract the number of counts of the signal is a Relativistic Breit-Wigner function modified by a Boltzmann factor to account for modification of the populated phase space in an hadronic heat bath [14]. The mean mass and width extracted from the fit are in a good agreement, within statistical errors, with the values reported by the Particle Data Group [5]. The losses due to decay, acceptance and reconstruction efficiency have to be corrected in order to extract the particle yield. These corrections are determined by means of GEANT simulations modeling the full detector response. The phase space distribution of $K^{* 0}$ was generated using the Siemens-Rasmussen formula [15] which describes a radially expanding equilibrated source characterized by a temperature $T$ and a radial expansion velocity $\beta$. The choice of the values of these parameters $(T=90 \mathrm{MeV}, \beta=0$ and 0.3$)$ is imposed by previous analysis measurements [1, 16] and the values of the radial flow are detailed below. The $K^{* 0}$ particles are embedded into an heavy-ion event calculated with the IQMD model [17]. The resulting output is subject to the same reconstruction procedure as for the experimental data. The distributions of simulated and measured event for all relevant quantities were carefully compared and found to be in good agreement [18, 19]. Finally, the reconstruction efficiency is determined by computing the ratio of reconstructed particles in the simulation to those initially embedded into the background events. The systematic error on the $K^{* 0}$ yield was evaluated in two steps. First the reconstruction efficiency was estimated from simulated $K^{* 0}$ with two values of the radial flow velocity $(\beta=0$ and $\beta=0.3)$. Then the $K^{* 0}$ signals were reconstructed under different sets of conditions on the relevant quantities used to perform the reconstruction, as for example, different cuts on $K^{+}$and $\pi^{-}$momentum. The use of a new variable in FOPI analysis like the $\theta^{*}$ [12], variations of histogram characteristics (bin-size) and different normalization areas used to fit the background were also taken into account for the systematic calculation.

$K_{S}^{0}$ were reconstructed in the $\pi^{+} \pi^{-}$decay channel (branching ratio $69.2 \%$ [5]) from its four momenta at the intersection point of the two pions tracks (Fig. 2). The combinatorial background was evaluated, as for the $K^{* 0}$ case, with the event mixing method. After background subtraction, a signal of about $10^{5}$ counts within a $\pm 2 \sigma$ window is extracted. The large statistics of reconstructed candidates allows to perform a bi-dimensional $\left(p_{t}, y^{0}\right)$ efficiency correction in order to extract the yield of $K^{0}$, where $y^{0}$ is the reduced rapidity $\left(y^{0}=\left(y^{l a b}-y^{c m}\right) / y^{c m}\right)$. The procedure used to simulate $K_{S}^{0}$ signals and $\mathrm{Al}+\mathrm{Al}$ collisions is the same as the one used for the $K^{* 0}$ particle. Decay products of $K_{L}^{0}$ cannot be measured with the FOPI apparatus. Since $K_{S}^{0}$ and $K_{L}^{0}$ are produced in equal amount (neglecting CP violation), the yield of $K^{0}$ corresponds to two times the one of $K_{S}^{0}$. The measured

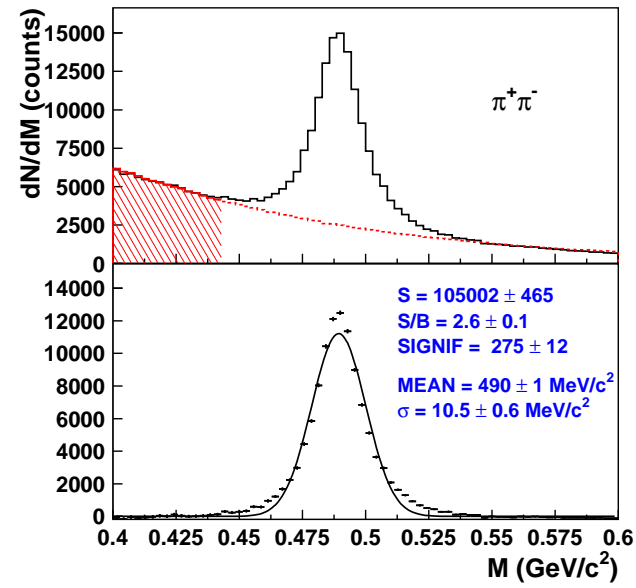

FIG. 2: (Color online) Invariant mass distribution of $\pi^{+} \pi^{-}$ pairs. The solid histogram and the dashed lines denote the data and the scaled mixed-event background, respectively (upper panel). The lower panel shows the signal after background subtraction. See caption of Fig. 1 for the abbreviations.

transverse mass spectra $\left(m_{t}=\sqrt{p_{t}^{2}+m_{0}^{2}}\right)$ of $K_{S}^{0}$ after efficiency correction are shown on the left side of Fig . B
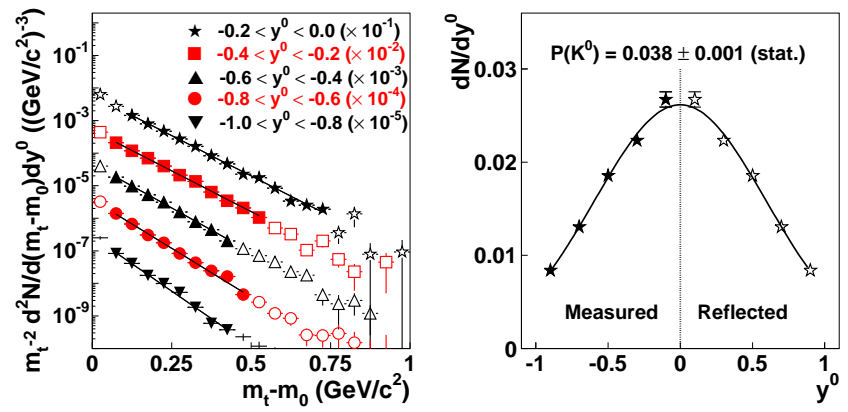

FIG. 3: (Color online) Left: Transverse mass distributions of $K_{S}^{0}$ mesons for different rapidity windows after efficiency corrections. The lines represent the Boltzmann function fits. The range of the data used to perform the adjustment are shown by full symbols. The values in parentheses denote scaling factors used for the plot. Right: Rapidity density distribution for $K^{0}$ where the open symbols are data points reflected with respect to mid-rapidity. The curve represents a Gaussian fit from which the total yield is extracted (see text for more details).

In order to extract the yield, we fit these distributions with a Boltzmann like function:

$$
\frac{1}{m_{t}^{2}} \frac{d^{2} N}{d\left(m_{t}-m_{0}\right) d y^{0}}=A \cdot \exp \frac{-\left(m_{t}-m_{0}\right)}{T_{B}}
$$

within a narrow window of rapidity $d y^{0}$. One can notice that, in the logarithmic representation, the measured 
transverse mass distributions exhibit a linear decrease with increasing $m_{t}-m_{0}$ and are described reasonably well by the Boltzmann function. The latter allows to extract the rapidity density distributions $d N / d y^{0}$ by integrating the fitted function from $m_{t}-m_{0}=0$ to $\infty$. The symmetry of the colliding system allows us to reflect the rapidity spectrum around mid-rapidity. The result, displayed in the right panel of Fig. 3, is fitted with a Gaussian function after applying correction factors by taking into account the branching ratio of $K_{S}^{0}(\times 100 / 69)$ and the unmeasured $K_{L}^{0}(\times 2)$. The integration of the Gaussian distribution allows to extract the $K^{0}$ yield per central (315 mb) collisions: $\mathrm{P}\left(K^{0}\right)=0.038 \pm 0.001$ (stat.) \pm 0.004 (syst.). The systematic errors of the measurement are obtained by using the same procedure applied for the $K^{* 0}$ particles. Finally, the $K^{* 0}$ to $K^{0}$ ratio is found to be:

$$
\frac{\mathrm{P}\left(K^{* 0}\right)}{\mathrm{P}\left(K^{0}\right)}=0.0315 \pm 0.006 \text { (stat.) } \pm 0.012 \text { (syst.). }
$$

Since for the $K^{* 0}$ resonance the efficiency correction is applied with a global factor due to the low statistics of the signal, such a ratio presents the advantage to cancel out in some extent the influence of the momentum distribution chosen for the simulated signals. The dependence on rapidity of the Boltzmann temperature $\left(T_{B}\right)$ of $K_{S}^{0}$ is found to be in good agreement with an isotropically emitting source $\left(T_{B}=T_{\text {eff }} / \cosh \left(y_{c . m .}\right)\right)$ [9], where $\mathrm{y}_{c . m}$. is the rapidity in the nucleus-nucleus center of mass. For the mid-rapidity region, the inverse slope reaches $T_{\text {eff }}=89 \pm 1$ (stat.) $\mathrm{MeV}$ and is consistent with the choice of $T=90 \mathrm{MeV}$ used to generate simulated signals.

These measurements complete the study of strangeness production in $\mathrm{Al}+\mathrm{Al}$ collisions at $1.9 \mathrm{~A} \mathrm{GeV}$ as presented in [1]. A summary of our experimental results is shown in Tab. I. The measured yield ratios are compared to the predictions by the UrQMD transport model [2, 20 22]. The UrQMD model allows to simulate heavy ion reac-

\begin{tabular}{|c|c|c|}
\hline Yield ratios & Data & UrQMD \\
\hline \hline$p / \pi^{-}$ & $5.3 \pm 2.4$ & 6.9 \\
\hline$\left(\Lambda+\Sigma^{0}\right) / \pi^{-}$ & $0.017 \pm 0.007$ & 0.018 \\
\hline$K^{0} /\left(\Lambda+\Sigma^{0}\right)$ & $0.66 \pm 0.13$ & 0.78 \\
\hline$\left(\Sigma^{*+}+\Sigma^{*-}\right) /\left(\Lambda+\Sigma^{0}\right)$ & $0.125 \pm 0.042$ & 0.177 \\
\hline$K^{* 0} / K^{0}$ & $0.032 \pm 0.013$ & 0.024 \\
\hline
\end{tabular}

TABLE I: Experimental ratios and predictions from UrQMD model. The error of the experimental results corresponds to the quadratic sum of statistical and systematic errors.

tions according to a N-body theory on an event by event basis. It is the only QMD-based model which allows the production of $\Sigma^{*}$ and $K^{*}$ at SIS energy 20, 21]. In this model, the Fermi motion (which is an important ingredient for particle production below threshold) is treated in the following way. The initial momenta of the nucleons are randomly chosen between 0 and the local ThomasFermi momentum [20]. The parameters used for the latter are set to their default values as reported in 23]. The model predictions are found to be in good agreement with the experimental ratios and their uncertainties. It is important to note that there is no explicit in-medium modifications of particle properties in this transport code. The good agreement deduced from the comparison of model predictions and data could be explained by a low nuclear density reached during the collision in the light $\mathrm{Al}+\mathrm{Al}$ system. A detailed discussion concerning the $\Sigma^{*}$ resonance production is provided in [1]. The dominant $K^{* 0}$ production process involved in the UrQMD model is the fusion of kaons and pions (70\%). The other processes to create $K^{* 0}$ involve reactions of non-strange resonances ( $\Delta$ and $N^{*}$ ) with baryons. The time dependence of these reactions exhibits a maximum around $7.5 \mathrm{fm} / c$. This coincides with the production time of $K^{+}$as predicted in [4]. This imply that the kaon-pion fusion occurs directly after kaon production.

In summary, we have presented new results on the $\mathrm{P}\left(K^{* 0}\right) / \mathrm{P}\left(K^{0}\right)$ strange mesons ratio in $\mathrm{Al}+\mathrm{Al}$ collisions at a beam energy of $1.9 \mathrm{~A} \mathrm{GeV}$. For the first time, the strange resonance $K^{* 0}$ was measured $800 \mathrm{MeV}$ below its production threshold in elementary reactions. The transverse mass spectra of $K_{S}^{0}$ were found to be consistent with a Boltzmann distribution. The experimental results on strange particles measured with the FOPI apparatus were compared to the predictions of the UrQMD transport model. The measurements are in good agreement with the model predictions where the dominant process to produce $K^{* 0}$ at sub-threshold energy is the fusion of kaons and pions. The measurement of the $K^{*}$ strange resonance in an heavier systems at sub-threshold energy could bring new informations on in-medium modification of particle properties at higher baryon density that can be reached at SIS energies and at the future FAIR facility.

We are grateful to M. Bleicher and S. Vogel for providing us model calculations and for intensive discussions. This work was supported by the German BMBF under Contract No. 06HD154, by the the National Research Foundation of Korea (NRF) under grant No. 2009-0070676, by the mutual agreement between GSI and IN2P3/CEA, by the Polish Ministry of Science and Higher Education under grant No. DFG/34/2007, by the Hungarian OTKA under grant No. 71989, within the Framework of the WTZ program (Project RUS 02/021), by DAAD (PPP D/03/44611) and by DFG (Projekt 446KOR-113/76/04). We have also received support by the European Commission under the 6th Framework Program under the Integrated Infrastructure on: Strongly Interacting Matter (Hadron Physics), Contract No. RII3CT-2004-506078. 
* Electronic address: lopez@clermont.in2p3.fr

[1] X. Lopez, N. Herrmann, P. Crochet et al. [FOPI Collaboration], Phys. Rev. C 76, 052203(R) (2007).

[2] M. Bleicher, Nucl. Phys. A 715, 85 (2003).

[3] C. Hartnack, H. Oeschler and J. Aichelin, Phys. Rev. Lett. 90, 102302 (2003).

[4] C. Hartnack, arXiv:nucl-th/0507002.

[5] C. Amsler et al., Phys. Lett. B 667, 1 (2008).

[6] A. Gobbi et al. [FOPI Collaboration], Nucl. Instrum. Meth. A 324, 156 (1993).

[7] J. Ritman [FOPI Collaboration], Nucl. Phys. Proc. Suppl. 44, 708 (1995).

[8] A. Andronic et al. [FOPI Collaboration], Nucl. Phys. A 679, 765 (2001).

[9] M. Merschmeyer, X. Lopez, N. Bastid, P. Crochet, N. Herrmann et al. [FOPI Collaboration], Phys. Rev. C 76, 024906 (2007).

[10] P. Crochet et al. [FOPI collaboration], Phys. Lett. B 486, 6 (2000).

[11] K. Wisniewski et al. [FOPI Collaboration], Eur. Phys. J.
A 9, 515 (2000).

[12] B. Hyppolite, PhD thesis, University Louis Pasteur, Strasbourg (2002).

[13] P. Danielewicz and G. Odyniec, Phys. Lett. B 157, 146 (1985).

[14] J. Adams et al. [STAR Collaboration], Phys. Rev. C 71, 064902 (2005).

[15] P.J. Siemens and J.O. Rasmussen, Phys. Rev. Lett. 42, 880 (1979).

[16] B. Hong et al. [FOPI Collaboration], Phys. Rev. C 57, 244 (1998) [Phys. Rev. C 58, 603 (1998)].

[17] C. Hartnack, R. K. Puri, J. Aichelin, J. Konopka, S. A. Bass, H. Stoecker and W. Greiner, Eur. Phys. J. A 1, 151 (1998).

[18] X. Lopez, PhD thesis, University of Clermont-Ferrand (2004).

[19] M. Merschmeyer, PhD thesis, University of Heidelberg (2004).

[20] S.A. Bass et al., Prog. Part. Nucl. Phys. 41, 225 (1998).

[21] M. Bleicher et al., J. Phys. G 25, 1859 (1999).

[22] S. Vogel (private communication).

[23] http://urqmd.org/documentation. 